\title{
The transitional gap transient AT 2018hso: new insights into the luminous red nova phenomenon ${ }^{\star}$
}

\author{
Y.-Z. Cai ${ }^{1,2}$, A. Pastorello ${ }^{2}$, M. Fraser ${ }^{3}$, S. J. Prentice ${ }^{4}$, T. M. Reynolds ${ }^{5}$, E. Cappellaro ${ }^{2}$, S. Benetti ${ }^{2}$, \\ A. Morales-Garoffolo ${ }^{6}$, A. Reguitti ${ }^{7,8,1}$, N. Elias-Rosa ${ }^{2}$, S. Brennan ${ }^{3}$, E. Callis ${ }^{3}$, G. Cannizzaro ${ }^{9,10}$, A. Fiore ${ }^{1,2}$, \\ M. Gromadzki ${ }^{11}$, F. J. Galindo-Guil ${ }^{12,13}$, C. Gall ${ }^{14}$, T. Heikkilä ${ }^{5}$, E. Mason ${ }^{15}$, S. Moran ${ }^{5,12}$, F. Onori ${ }^{16}$, \\ A. Sagués Carracedo ${ }^{17}$, and G. Valerin ${ }^{1,2}$ \\ (Affiliations can be found after the references)
}

Received 20 September 2019 / Accepted 6 November 2019

\begin{abstract}
Context. The absolute magnitudes of luminous red novae (LRNe) are intermediate between those of novae and supernovae (SNe), and show a relatively homogeneous spectro-photometric evolution. Although they were thought to derive from core instabilities in single stars, there is growing support for the idea that they are triggered by binary interaction that possibly ends with the merging of the two stars.

Aims. AT 2018hso is a new transient showing transitional properties between those of LRNe and the class of intermediate-luminosity red transients (ILRTs) similar to SN 2008S. Through the detailed analysis of the observed parameters, our study supports that it actually belongs to the LRN class and was likely produced by the coalescence of two massive stars.

Methods. We obtained ten months of optical and near-infrared photometric monitoring, and 11 epochs of low-resolution optical spectroscopy of AT 2018hso. We compared its observed properties with those of other ILRTs and LRNe. We also inspected the archival Hubble Space Telescope (HST) images obtained about 15 years ago to constrain the progenitor properties.

Results. The light curves of AT 2018hso show a first sharp peak (reddening-corrected $M_{r}=-13.93 \mathrm{mag}$ ), followed by a broader and shallower second peak that resembles a plateau in the optical bands. The spectra dramatically change with time. Early-time spectra show prominent Balmer emission lines and a weak [Ca II] doublet, which is usually observed in ILRTs. However, the strong decrease in the continuum temperature, the appearance of narrow metal absorption lines, the great change in the $\mathrm{H} \alpha$ strength and profile, and the emergence of molecular bands support an LRN classification. The possible detection of a $M_{\mathrm{I}} \sim-8 \mathrm{mag}$ source at the position of AT 2018hso in HST archive images is consistent with expectations for a pre-merger massive binary, similar to the precursor of the 2015 LRN in M101.

Conclusions. We provide reasonable arguments to support an LRN classification for AT $2018 \mathrm{hso}$. This study reveals growing heterogeneity in the observables of LRNe than has been thought previously, which is a challenge for distinguishing between LRNe and ILRTs. This suggests that the entire evolution of gap transients needs to be monitored to avoid misclassifications.
\end{abstract}

Key words. binaries: close - stars: winds, outflows - stars: massive - supernovae: individual: AT 2018hso - supernovae: individual: AT 2017jfs supernovae: individual: NGC4490-20110T1

\section{Introduction}

Modern all-sky surveys are discovering stellar transients with intrinsic luminosities that lie in the middle between those of core-collapse supernovae (CC SNe) and classical novae $(-15 \leqslant$ $\left.M_{\mathrm{V}} \leqslant-10 \mathrm{mag}\right)$. These are collectively known as "gap transients" (e.g. Kasliwal 2012; Pastorello \& Fraser 2019) and include intermediate-luminosity red transients (ILRTs; e.g. Botticella et al. 2009; Thompson et al. 2009; Berger et al. 2009) and luminous red novae(LRNe; e.g. Munari et al.2002; Tylenda2005; Williams et al. 2015; Goranskij et al. 2016; Lipunov et al. 2017).

The single-peaked light curves of ILRTs resemble those of faint SNe IIP or IIL. Their spectra show prominent Balmer emission features, along with weak Fe II, Na I D, and Ca II lines. In particular, the [Ca II] doublet feature, which is prominent in all phases, is a typical signature of ILRTs. These transients are physically consistent with electron-capture-induced supernova

\footnotetext{
* The light curves and the reduced spectra are only available at the CDS via anonymous ftp to cdsarc.u-strasbg. fr (130.79.128.5) or via http://cdsarc.u-strasbg.fr/viz-bin/cat/J/A+A/632/
} L6
(EC SN) explosions from super-asymptotic giant branch stars (S-AGB; see, e.g. Pumo et al. 2009). Well-studied objects are SN 2008S (e.g. Botticella et al. 2009), NGC 300 OT2008-1 (e.g. Humphreys et al. 2011), and AT 2017be (Cai et al. 2018).

The LRNe compose a distinct group of gap transients, which usually display double-peaked light curves (e.g. Kankare et al. 2015) and a distinct spectral evolution with time. In particular, a forest of narrow metal lines in absorption are detected in the spectra during the second light-curve maximum, and broad molecular absorption bands (e.g. TiO) are observed at late epochs (e.g. Kamiński et al. 2009; Mason et al. 2010; Barsukova et al. 2014). LRNe are very likely produced by extreme interaction in a close binary system that leads to the ejection of the common envelope (CE). The final outcome is likely a merger (e.g. Tylenda et al. 2011; Kochanek et al. 2014; Pejcha et al. 2016).

While most LRNe have a robust classification, it is occasionally difficult to distinguish them from ILRTs (see e.g. the controversial cases of M85-2006OT1 and PTF 10fqs; Kulkarni et al. 2007; Kasliwal et al. 2011). In this context, we report the study of AT 2018hso, which shows hybrid spectro-photometric properties that challenge the existing paradigm of LRNe. 


\section{Object information}

The transient AT 2018hso (also known as ZTF18acbwfza) was discovered on 2018 October 31.53 (UT) by the Zwicky Transient Facility (ZTF; De et al. 2018). Its coordinates are $\mathrm{RA}=11^{\mathrm{h}} 33^{\mathrm{m}} 51.96^{\mathrm{s}}$, Dec $=+53^{\circ} 07^{\prime} 07.10^{\prime \prime}$ [J2000], 24.9" south, 23.7" east of the core of the face-on late-type galaxy NGC 3729.

The distances to NGC 3729 reported in the NASA/IPAC Extragalactic Database (NED) are based on the Tully-Fisher method (e.g. Willick et al. 1997; Tully et al. 2009) and range from 21.10 to $21.88 \mathrm{Mpc}$. The kinematic distance corrected for Virgo infall and obtained adopting a standard cosmology $\left(H_{0}=73 \mathrm{~km} \mathrm{~s}^{-1} \mathrm{Mpc}^{-1}, \Omega_{\mathrm{M}}=0.27\right.$, and $\left.\Omega_{\Lambda}=0.73\right)$ is $d_{\mathrm{k}}=$ $20.80 \pm 1.5 \mathrm{Mpc}$ (Mould et al. 2000) and agrees well with TullyFisher estimates. We therefore hearafter adopt the weighted average of the above values, $d=21.26 \pm 0.56 \mathrm{Mpc}$, as distance to NGC 3729. This provides a distance modulus $\mu=31.64 \pm$ 0.06 mag.

The Galactic reddening at the coordinates of AT 2018hso is very low, $E(B-V)_{\mathrm{Gal}}=0.01 \mathrm{mag}$ (Schlafly \& Finkbeiner 2011). Early spectra have modest resolution and a limited signal-tonoise ratio $(\mathrm{S} / \mathrm{N})$, while late-time spectra are affected by a forest of absorption metal lines. For an indicative estimate of the host galaxy reddening, however, we averaged the measured equivalent widths (EWs) of the $\mathrm{Na}$ I $\mathrm{D}$ absorption in two early spectra (at -3.8 and $+11.3 \mathrm{~d}$ ) and obtained $\mathrm{EW}=1.8 \pm 0.5 \AA$. Following the relation in Turatto et al. (2003), we obtain a host galaxy reddening $E(B-V)_{\text {Host }}=0.29 \pm 0.08 \mathrm{mag}$. The total line-of-sight reddening is therefore $E(B-V)_{\text {Total }}=0.30 \pm 0.08 \mathrm{mag}$.

\section{Photometry}

We started the monitoring of AT 2018hso soon after its discovery, and the follow-up campaign lasted $\sim 300 \mathrm{~d}$. The photometric data were reduced using the SNoOpy pipeline, and the resulting point-spread function (PSF) fitting optical and near-IR (NIR) apparent magnitudes are reported in Tables A.1 and A.2. The multi-band light curves are shown in the top panel of Fig. 1. The $r$-band light curve rises to the first maximum (on MJD $=58431.0 \pm 1.0)$ in $\sim 8 \mathrm{~d}$. The peak magnitude is $r \sim$ $18.40 \mathrm{mag}$ (reddening-corrected $M_{r} \sim-13.93 \mathrm{mag}$ ). After this, the $r$-band light curve first declines $(\sim 5.3 \mathrm{mag} / 100 \mathrm{~d})$, and then slowly rises again to a second, fainter maximum at around $80 \mathrm{~d}$ (on MJD $\approx 58515$ ). The magnitude of the second peak is $r \sim$ $19.57 \mathrm{mag}$ (reddening-corrected $M_{r} \sim-12.76 \mathrm{mag}$ ). A monotonic decline follows, which is initially slow $(\sim 1.7 \mathrm{mag} / 100 \mathrm{~d})$, but later rapidly increases to about $3.8 \mathrm{mag} / 100 \mathrm{~d}$. While the $i$ and $z$ bands have a similar evolution as the $r$ band, the bluer bands $(B, g$, and $V)$ show a sort of plateau. The few $u$-band detections show a roughly linear decline of $\sim 15.7 \mathrm{mag} / 100 \mathrm{~d}$. The NIR light curves resemble those in the red bands, showing a more evident long-duration $(\sim 150 \mathrm{~d})$ second maximum, followed by a moderate decline $(\sim 1.3 \mathrm{mag} / 100 \mathrm{~d})$ starting from $\sim 190 \mathrm{~d}$.

We compare the $r$-band absolute light curve of AT 2018hso with some well-followed gap transients, two ILRTs (SN 2008S and AT 2017be), and two LRNe (NGC4490-2011OT1 and AT 2017jfs) in Fig. 1 (bottom panel). Their maximum absolute magnitudes range from -12 to $-15.5 \mathrm{mag}$. ILRTs show a singlepeaked SN-like light curve, and the LRNe have double-peaked light curves. We note that AT 2018hso reveals a transitional light curve, between ILRTs and LRNe, with a first sharp blue peak followed by a much shallower and broader red peak.

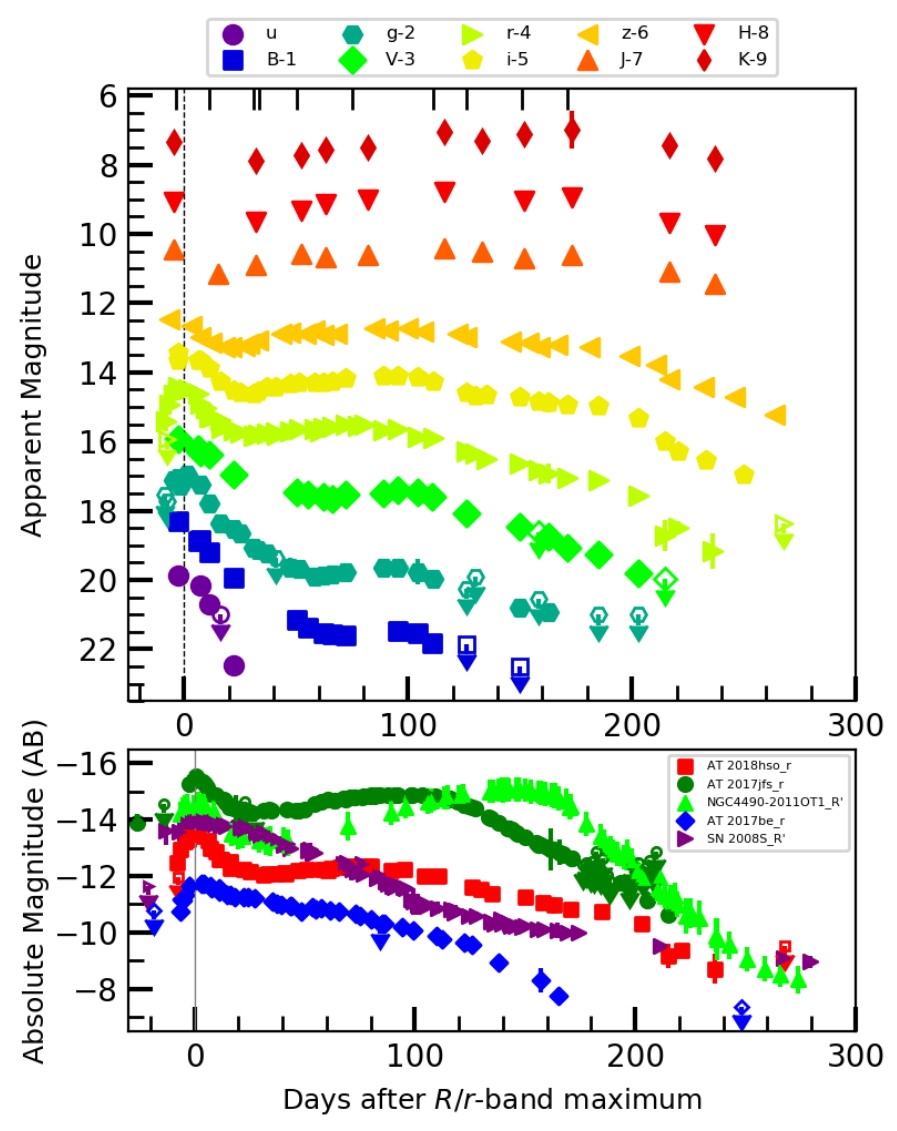

Fig. 1. Top: BVugrizJHK light curves of AT 2018hso. The epochs of optical spectra are also marked. Bottom: sloan $r$-band absolute light curves of AT 2018hso, two ILRTs (SN 2008S and AT 2017be), and two LRNe (NGC4490-2011OT1 and AT 2017jfs).

\section{Spectroscopy}

Our spectroscopic campaign spans a period of eight months, from $\sim-4$ to $+233 \mathrm{~d}$, of the AT 2018 hso evolution. We collected ten epochs of spectroscopic observations with the $2.56 \mathrm{~m}$ Nordic Optical Telescope (NOT) equipped with ALFOSC. In addition, through approved Director's Discretionary Time (DDT, PI: A. Morales-Garoffolo), a very late spectrum was obtained on 2019 June 29 with the $10.4 \mathrm{~m}$ Gran Telescopio Canarias (GTC) plus OSIRIS (see details in Table A.3). The spectra were processed following standard tasks in IRAF. The spectral evolution of AT 2018hso is presented in Fig. 2, and the comparison with ILRTs AT 2017be and SN 2008S, and LRN AT 2017jfs is shown in Fig. 3. Prominent spectral lines are marked in Fig. 3.

The spectral evolution of AT 2018hso is characterised by three distinct phases, in analogy with the behaviour of other LRNe (Pastorello et al. 2019a,b). At early epochs (until 30 d), the spectra show a blue continuum, with prominent Balmer lines, along with a number of Fe II features, $\mathrm{Na}$ I D $(\lambda=5890,5896 \AA$ ), and Ca II emission lines (see Fig. 2 and Fig. 3, top panel). The temperature inferred from the spectral continuum, assuming a black-body spectral energy distribution (SED), decreases from $8800 \pm 800 \mathrm{~K}$ (at $-3.8 \mathrm{~d}$ ) to $6800 \pm 600 \mathrm{~K}$ (at $+11.3 \mathrm{~d}$ ). We measured the full width at half-maximum (FWHM) velocity of $\mathrm{H} \alpha$ through a Lorentzian fit, and obtained $v_{\mathrm{FWHM}} \sim 500 \mathrm{~km} \mathrm{~s}^{-1}$ (accounting for the instrumental resolution, see Table A.3). This early-time velocity is similar to those measured for LRNe, but also some ILRTs, such as AT 2017be (also fitted by Lorentzian 


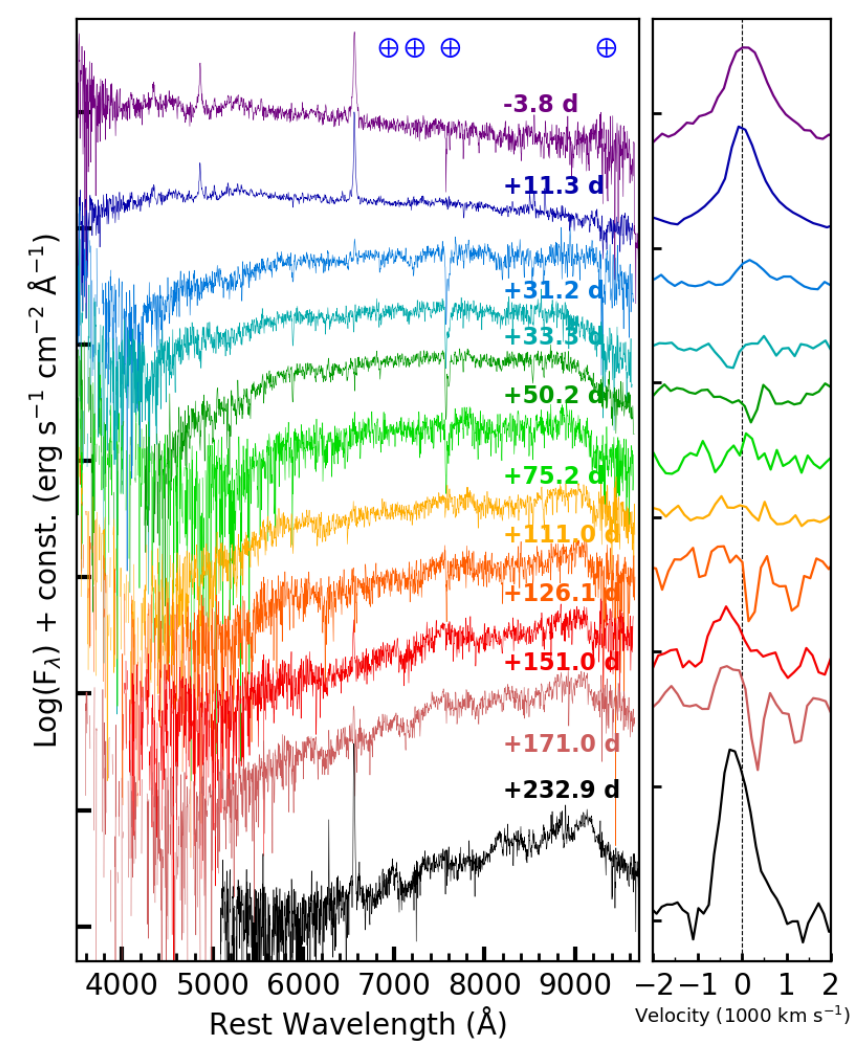

Fig. 2. Left: spectral evolution of AT 2018hso from -3.8 to $+232.9 \mathrm{~d}$. Right: evolution of the $\mathrm{H} \alpha$ profile in the velocity space. The vertical dotted line marks the rest velocity. All spectra are redshift corrected $(z=0.003536$, see NED). The phases are from the $r$-band maximum.

function, $\sim 500-800 \mathrm{~km} \mathrm{~s}^{-1}$; Cai et al. 2018). A prominent Ca II NIR triplet $(\lambda=8498,8542,8662 \AA)$ is detected in emission in the AT 2018hso spectra, along with a very weak [Ca II] doublet $(\lambda=7291,7328 \AA)$. We remark that a prominent [Ca II] is a ubiquitous feature in the spectra of ILRTs at all phases (Cai et al. 2018). In contrast, it has never been securely identified in LRN spectra so far. Ca II H\&K $(\lambda=3934,3968 \AA)$ is not clearly detected, but the spectra are very noisy below $4000 \AA$. All of this makes early spectra of AT 2018hso similar to those of ILRTs (see Fig. 3).

During the rise to the second peak (from $\sim 30 \mathrm{~d}$ to $120 \mathrm{~d}$ ), the spectra experience a dramatic evolution. The continuum becomes redder: the continuum temperature decreases from $\sim 4500$ to $3000 \mathrm{~K}$. Thus, the spectra resemble those of cool stars ( $\mathrm{K}$ to early-M types). $\mathrm{H} \alpha$ is marginally detected at $+31.2 \mathrm{~d}$, and the emission component disappears until $+111.0 \mathrm{~d}$. In spectra with the highest $\mathrm{S} / \mathrm{N}$, we identify metal lines (Fe II, Ba II, and $\mathrm{Sc}$ II) in absorption. We note that these lines are also detected in ILRTs (Humphreys et al. 2011). These features, in particular the $\mathrm{Ba}$ II lines, are frequently detected in stellar outbursts or CC SN explosions, and originate in regions where the gas temperature is lower (Pastorello et al. 2004). As a remarkable note, the [Ca II] doublet is no longer detected. In contrast with early spectra, the NIR Ca II triplet is now seen in absorption. Ca II NIR triplet is formed by radiative de-excitation from $4 \mathrm{p}^{2} \mathrm{P}_{1 / 2,3 / 2}$ to $3 \mathrm{~d}^{2} \mathrm{D}_{3 / 2,5 / 2}$ levels (Mallik 1997), while the [Ca II] doublet originates in lowdensity gas with a critical density of about $10^{7} \mathrm{~cm}^{-3}$, which is related to the metastable $3 \mathrm{~d}^{2} \mathrm{D}$ level (Ferland \& Persson 1989). The strong change in these lines indicates that the gas densities evolve with time. At this phase, the spectra of AT 2018hso

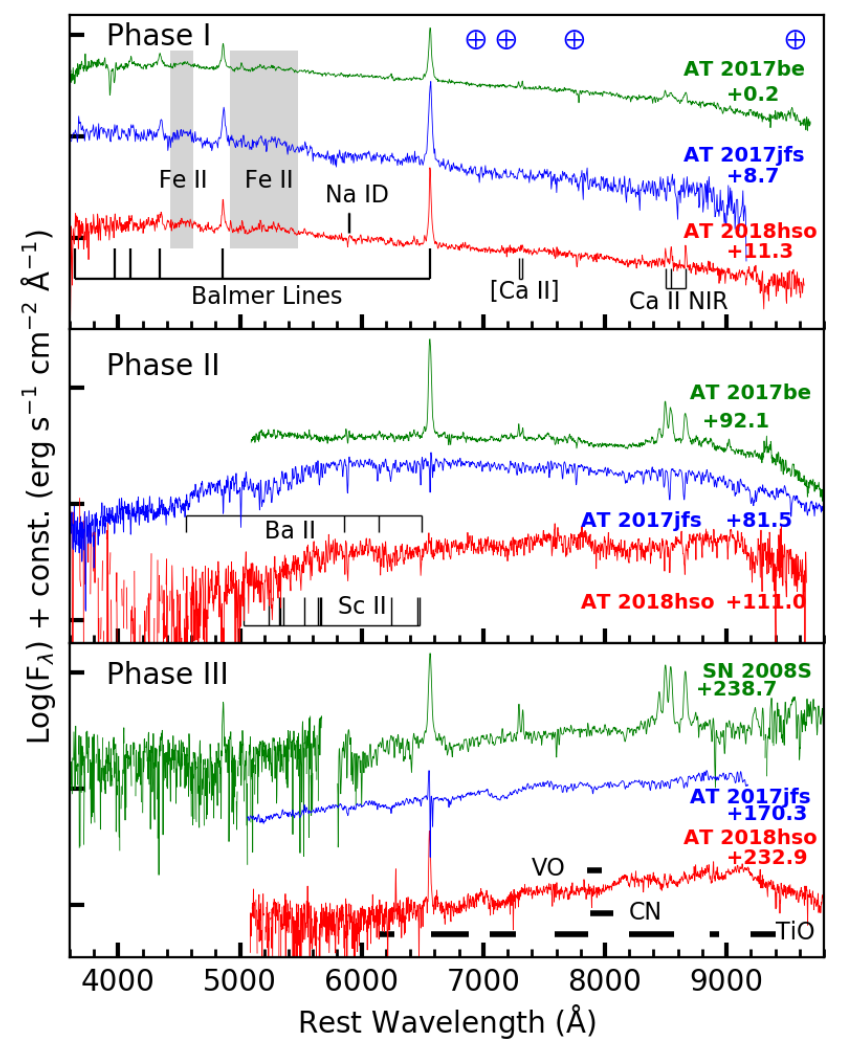

Fig. 3. Spectral comparison of AT 2018hso with LRN AT 2017jfs, and ILRTs AT 2017be and SN 2008S, with line identification. The spectra are selected at three representative epochs: phase I $(0$ to $+10 \mathrm{~d}$; top panel), phase II $(95 \pm 15 \mathrm{~d}$; middle panel), and phase III ( $>170 \mathrm{~d}$; bottom panel). Phases are from the $R / r$-band maximum. All spectra are corrected for redshift and reddening.

are reminiscent of LRNe and are clearly different from those observed in ILRTs (see Fig. 3, middle panel).

At very late times (over four months after the peak), the spectra of AT $2018 \mathrm{hso}$ become even redder $(T=2050 \pm 200 \mathrm{~K}$ at +232.9 d) and resemble those of a late-M star. $\mathrm{H} \alpha$ becomes prominent again, with $v_{\mathrm{FWHM}} \sim 370 \mathrm{~km} \mathrm{~s}^{-1}$ at $+232.9 \mathrm{~d}$, as obtained by fitting a Gaussian function because the Lorentzian wings are no longer visible in the line profile. In addition, the $\mathrm{H} \alpha$ emission peak appears blue-shifted by about 300 to $400 \mathrm{~km} \mathrm{~s}^{-1}$ at $\sim 150$ to $230 \mathrm{~d}$, respectively ${ }^{1}$ (see the right panel of Fig. 2). This is reminiscent of very late spectra of AT 2017jfs and NGC44902011OT1 and supports an LRN classification for AT 2018hso. The asymmetries in the $\mathrm{H} \alpha$ profile suggest that the geometry of the emitting region is aspherical. Alternatively, it might signify new dust formation that obscures the receding material, or both scenarios might be true (Smith et al. 2016; Pastorello et al. 2019 b). In addition, very late spectra of AT 2018hso show broad molecular bands, such as TiO and possibly VO and CN (Fig. 3, bottom panel). This is a key feature that allows us to distinguish LRNe from ILRTs (Pastorello et al. 2019b). Following the interpretation of Kamiński et al. (2009), we may speculate that the TiO molecular bands of AT 2018hso originate in the warm photosphere $(T>2000 \mathrm{~K})$, but also in the cold outflowing material $(T \sim 500 \mathrm{~K})$ with a velocity about $-3-400 \mathrm{~km} \mathrm{~s}^{-1}$, where it is similarly likely that VO features form.

1 Narrow absorptions are found at $+126.1 \mathrm{~d}$ and $+171.0 \mathrm{~d}$, and are likely due to over-subtraction of contaminating background. 


\section{Spectral energy distribution}

We investigated the SED evolution of AT 2018hso using photometric data. The SEDs were selected at a few representative epochs throughout the whole monitoring period. The SEDs were fitted with a single Planck function, assuming that the photosphere radiates as a black-body. At early phases, before the blue peak, the object was only occasionally observed. When individual broad-band data were not available, the missing point was estimated by interpolating the data at two consecutive epochs, or by extrapolating from the last available observation. All of this implies larger errors. At 30-110 d, the optical plus NIR SEDs are well reproduced with a single black-body. Instead, although at later phases blue-band $(u, B, g, V)$ data are not available, a single black-body does not appear to accurately fit the observed SEDs. This is even more evident during the final steep lightcurve decline (at $\sim 210 \mathrm{~d}$ ). This is suggestive of a second blackbody component peaking at much longer wavelengths (mid- to far-IR), although it cannot be confirmed because the photometric coverage is incomplete.

The temporal evolution of the black-body temperature is shown in Fig. 4 (top panel). The temperature rapidly declines from $\sim 8000 \mathrm{~K}$ at maximum to $\sim 4000 \mathrm{~K}$ at about $30 \mathrm{~d}$. Then, it declines more slowly to $\sim 3000 \mathrm{~K}$ until $180 \mathrm{~d}$. A steeper temperature drop (to around $2500 \mathrm{~K}$ ) follows at $\sim 210 \mathrm{~d}$. The inferred bolometric luminosity, obtained by integrating the SEDs throughout the full electromagnetic spectrum, is shown in the middle panel of Fig. 4. The luminosity at peak is about $10^{41} \mathrm{erg} \mathrm{s}^{-1}$, then it rapidly declines to a minimum $(L \sim 3.2 \times$ $\left.10^{40} \mathrm{erg} \mathrm{s}^{-1}\right)$, rises again to the second, red maximum $(L \sim 4.4 \times$ $\left.10^{40} \mathrm{erg} \mathrm{s}^{-1}\right)$, and finally rapidly drop to $L \sim 1.6 \times 10^{40} \mathrm{erg} \mathrm{s}^{-1}$. The inferred radius, obtained through the Stefan-Boltzmann law ( $L=4 \pi R^{2} \sigma T^{4}$; where $\sigma$ is the Stefan-Boltzmann constant), is shown in the bottom panel of Fig. 4. The radius remains roughly constant at $\sim 2 \times 10^{14} \mathrm{~cm}$ (almost $2900 R_{\odot}$ ) during the first $\sim 30 \mathrm{~d}$, then it rapidly increases to $\sim 5.3 \times 10^{14} \mathrm{~cm}$ (exceeding $7600 R_{\odot}$ ) at $\sim 80 \mathrm{~d}$, to increase by a modest amount later. A significant increase in radius is observed after $\sim 210 \mathrm{~d}$ (although it is affected by large uncertainties), and exceeds $\sim 7.2 \times 10^{14} \mathrm{~cm}$ $\left(R \sim 10350 R_{\odot}\right)$.

These parameters of AT 2018hso are compared with those of a few ILRTs and LRNe in Fig. 4. While the temperature evolution is not relevant for distinguishing the two classes, the bolometric light curve and the radius evolution are markedly different in LRNe and ILRTs. Specifically, ILRT light curves decline monotonically after maximum, while LRN light curves reveal a second bolometric peak. The difference between LRNe and ILRTs is even more evident in the radius evolution. While in ILRTs the radius at the photosphere of the hot black-body component declines by a factor of two (see Botticella et al. 2009), it increases by one order of magnitude during the LRN evolution, with the strongest increase during the rise to the second photometric peak. A further increase in the value of the radius is observed at very late phases, during the final steep optical decline. This is also observed in AT 2017jfs at the time of a NIR brightening. In this phase, the $\mathrm{H} \alpha$ profile is blue-shifted and the spectra show molecular bands. The different light curves and radius evolution in ILRTs and LRNe suggest that the two classes of gap transients are regulated by different physical mechanisms. The outer layers become optically thin with the expansion, and the photosphere recedes into deeper ejecta, which together probably causes the ILRT radius to contract after the peak. The more complex evolution of the LRN radii, as previously noted by Blagorodnova et al. (2017) for M101-2015OT1, is more difficult

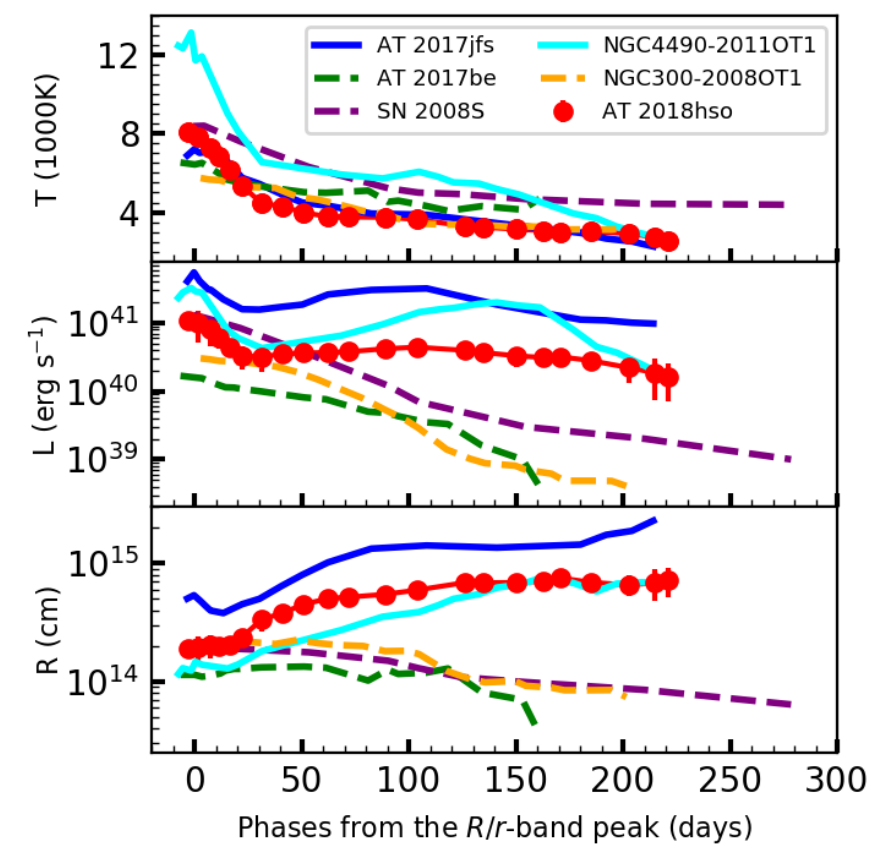

Fig. 4. Comparison of AT 2018hso with three ILRTs (dashed lines): SN 2008S, NGC300-2008OT1 (Humphreys et al. 2011), and AT 2017be, and two LRNe (solid lines): AT 2017jfs, NGC4490-2011OT1. Top: black-body temperature evolution. Middle: bolometric light curves. Bottom: evolution of the photospheric radii.

to explain. In particular, while M31-2015LRN (MacLeod et al. 2017) showed a contraction of the radius at late phases, which is consistent with a receding photosphere with expansion, brighter LRNe are characterised by at least two phases with an almost constant radius. These phases occur soon after the first and the second peaks. After the first peak, the opacity in the expanding ejecta decreases and thus the radius in mass coordinate decreases. When these ejecta reach the material that is expelled during the CE phase, this gas is initially heated, and a second photosphere forms far out, which causes the radius to increase. The ejecta plus CE-shocked region expands and remains optically thick, therefore the photospheric radius grows.

\section{Nature of AT 2018hso}

The transient AT 2018hso shows transitional light curves between ILRTs and LRNe, and early spectra similar to those of ILRTs. However, we favour an LRN classification for the following reasons:

- The spectra experience a significant temporal evolution. Early-time spectra resemble those of ILRTs. Nonetheless, the LRN scenario for AT 2018hso is supported by the vanishing [Ca II] doublet with time, the tremendous change of $\mathrm{H} \alpha$ profile, and the late appearance of molecular bands.

- In analogy to other LRNe, it peaks at an absolute magnitude of reddening-corrected $M_{r} \sim-13.93 \mathrm{mag}$ after a fast rise that lasts about $8 \mathrm{~d}$.

- The SED and the evolution of the photospheric radius are similar to those observed in other LRNe.

- The decay time from peak luminosity $\left(L_{\mathrm{peak}} \approx 10^{41} \mathrm{erg} \mathrm{s}^{-1}\right)$ to $0.5 L_{\text {peak }}$ is around $15 \mathrm{~d}$. This is located near the LRN region in the luminosity versus $\tau_{0.5 \mathrm{dex}}$ diagram of Pastorello \& Fraser (2019, see their Fig. 1).

- The outflow velocity of AT 2018 hso is about $400 \mathrm{~km} \mathrm{~s}^{-1}$, which places it slightly below NGC4490-2011OT1 in the 
$L_{\text {peak }}$ versus $v_{\text {out }}$ diagram of Mauerhan et al. (2018), but it is still well aligned with other LRNe.

To add further support to the LRN classification, we inspected HST images obtained from the Hubble Legacy Archive. The absolute magnitude of the recovered progenitor $\left(M_{\mathrm{I}} \sim-8 \mathrm{mag}\right.$; see Appendix A) is at least plausibly consistent with a pre-merger system that has started to brighten about $15 \mathrm{yr}$ before the coalescence, similar to what was observed in the 2015 LRN in M101 (Blagorodnova et al. 2017). However, in the absence of more precise astrometry and at the spatial resolution of our data at the distance of NGC 3729, we cannot exclude that this is a cluster or an unrelated source. Late-time observations after the merger has faded and cooled will reveal if this was the progenitor system, as we would expect this source to become dust enshrouded, disappearing (or at least fading) in the optical. Pastorello et al. (2019b) studied a large sample of LRNe and supported for them a stellar merger scenario in a binary system following the ejection of a CE (e.g. Pejcha et al. 2016, 2017; MacLeod et al. 2017). Kochanek et al. (2014) and Smith et al. (2016) suggested that energetic LRN events, such as NGC44902011OT1, are likely the outcome of massive $\left(10 \leq M \leq 50 M_{\odot}\right)$ binary mergers. In this context, because a luminous source is reliably detected in AT 2018hso and it is only marginally fainter than NGC4490-20110T1, its progenitor system was very likely quite massive (Kochanek et al. 2014) .

From the study of the spectro-photometric evolution of AT 2018hso and the possible detection of the progenitor system in archive HST images, we provided reasonable arguments for classifying AT 2018hso as an LRN, although with somewhat peculiar characteristics. So far, only two other objects have controversial ILRT/LRN classifications to our knowledge. Although M85-2006OT1 was proposed to be an LRN (Kulkarni et al. 2007; Rau et al. 2007), Pastorello et al. (2007) questioned it, and an ILRT scenario is still plausible from observational arguments (e.g. Kochanek et al. 2014). Another gap transient, AT 2019abn, was classified as an ILRT by Jencson et al. (2019) on the basis of follow-up observations lasting $\sim 110 \mathrm{~d}$ and a detailed study of the progenitor in pre-outburst stage. However, the initial weakness of the [Ca II] doublet and its later disappearance (see Fig. 5) are reminiscent of LRNe. The lack of strong $\mathrm{TiO}$ and $\mathrm{VO}$ bands in the optical spectra will provide further support to the ILRT classification. All of this suggests the existence of a grey zone in our ability to distinguish the different classes of gap transients.

We have limited knowledge on ILRTs and LRNe because the number of discoveries is still modest and the data sets are incomplete. Well-sampled light curves in a wide range of wavelengths, high-resolution spectroscopy, and detailed modelling are crucial to clarify the nature of these two families of gap transients. The support of future-generation instruments such as the Large Synoptic Survey Telescope (LSST Science Collaboration 2009) and the Wide Field Infrared Survey Telescope (WFIRST; Spergel et al. 2015) is essential to increase the number of wellmonitored gap transients, which in turn is crucial to fine-tune existing theoretical models.

Acknowledgements. We thank the referee for his/her helpful comments/suggestions that have improved this Letter. We thank K. Maguire, X-F. Wang for useful discussions. Y-Z.C is supported by the China Scholarship Council (No. 201606040170). KM and SJP are supported by H2020 ERC grant no. 758638. MF is supported by a Royal Society - Science Foundation Ireland University Research Fellowship. SB and AF are partially supported by PRIN-INAF 2017 of Toward the SKA and CTA era: discovery, localization, and physics of transient sources.(PI: M. Giroletti). T.R. acknowledges the financial support of the Jenny and Antti Wihuri and the Vilho, Yrjö and Kalle Väisälä foundations. AR acknowledges financial support by the "Millennium Institute

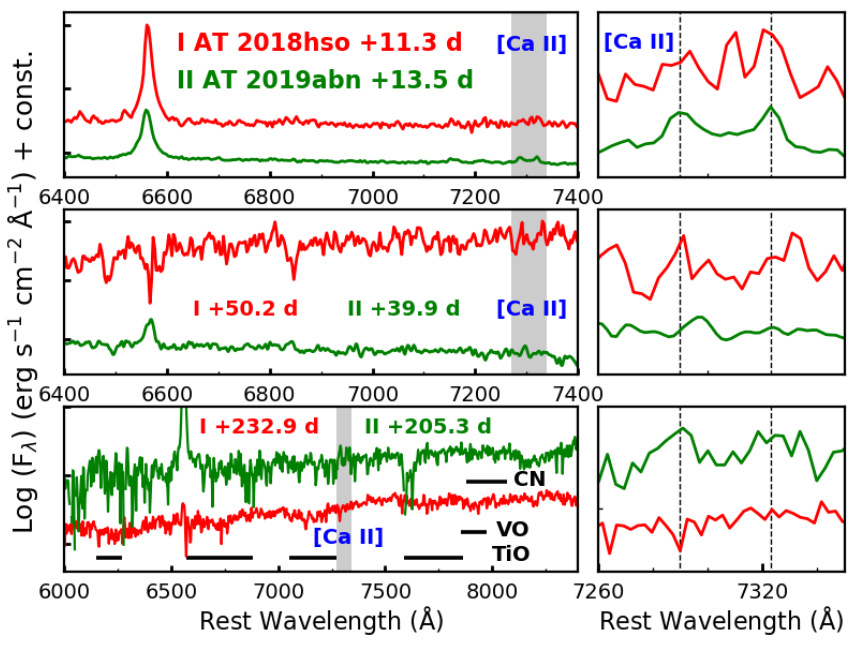

Fig. 5. Spectral comparison of AT 2018hso and AT 2019abn. Left: spectral evolution at three representative phases: $\sim 12 \pm 1 \mathrm{~d}$ (top), $\sim 45 \pm 5 \mathrm{~d}$ (middle), and $\geq 200 \mathrm{~d}$ (bottom). The molecular bands are identified in the left bottom panel. The [Ca II] doublet is marked with a shaded grey region in the three left panels. Right: zoom-in of the [Ca II] doublet profile at the same phases. Phases are from their $r$-band maximum.

of Astrophysics (MAS)" of the Iniciativa Cientìfica Milenio. CG acknowledges support by the VILLUM FONDEN research grant (project number 00025501) and Investigator grant (project number 16599). MG is supported by the Polish NCN MAESTRO grant 2014/14/A/ST9/00121. FO acknowledges the support of the H2020 Hemera program, grant agreement No 730970. The Nordic Optical Telescope (NOT), operated by the NOT Scientific Association at the Spanish Observatorio del Roque de los Muchachos of the Instituto de Astrofisica de Canarias. Observations from the NOT were obtained through the NUTS1 and NUTS2 collaboration which are supported in part by the Instrument Centre for Danish Astrophysics (IDA). The data presented here were obtained (in part) with ALFOSC, which is provided by the Instituto de Astrofisica de Andalucia (IAA) under a joint agreement with the University of Copenhagen and NOTSA. The Liverpool Telescope is operated on the island of La Palma by Liverpool John Moores University in the Spanish Observatorio del Roque de los Muchachos of the Instituto de Astrofisica de Canarias with financial support from the UK Science and Technology Facilities Council. Based on observations made with the GTC telescope in the Spanish Observatorio del Roque de los Muchachos of the Instituto de Astrofssica de Canarias, under Director's Discretionary Time (GTC2019-127; PI: A. Morales-Garoffolo). This research makes use of Lasair data (https://lasair.roe.ac.uk/), which is supported by the UKRI Science and Technology Facilities Council and is a collaboration between the University of Edinburgh (grant ST/N002512/1) and Queen's University Belfast (grant ST/N002520/1) within the LSST:UK Science Consortium. IRAF is written and supported by the National Optical Astronomy Observatories (NOAO) in Tucson, Arizona. NOAO is operated by the Association of Universities for Research in Astronomy (AURA), Inc. under cooperative agreement with the National Science Foundation. This research has made use of the NASA/IPAC Extragalactic Database (NED), which is operated by the Jet Propulsion Laboratory, California Institute of Technology, under contract with the National Aeronautics and Space Administration. SNOoPy is a package developed by E. Cappellaro which performs photometry of point-like sources in complex environments using PSF-fitting and template subtraction methods. A package description can be found at http://sngroup.oapd.inaf.it/snoopy.html.

\section{References}

Barsukova, E. A., Goranskij, V. P., Valeev, A. F., \& Zharova, A. V. 2014, Astrophys. Bull., 69, 67

Berger, E., Soderberg, A. M., Chevalier, R. A., et al. 2009, ApJ, 699, 1850

Blagorodnova, N., Kotak, R., Polshaw, J., et al. 2017, ApJ, 834, 107 Botticella, M. T., Pastorello, A., Smartt, S. J., et al. 2009, MNRAS, 398, 1041 Cai, Y.-Z., Pastorello, A., Fraser, M., et al. 2018, MNRAS, 480, 3424 De, K., Fremling, C., Miller, A. A., et al. 2018, ATel, 12162 Ferland, G. J., \& Persson, S. E. 1989, ApJ, 347, 656

Goranskij, V. P., Barsukova, E. A., Spiridonova, O. I., et al. 2016, Astrophys. Bull., 71, 82

Humphreys, R. M., Bond, H. E., Bedin, L. R., et al. 2011, ApJ, 743, 118 
Jencson, J. E., Adams, S. M., Bond, H. E., et al. 2019, APJ, 880, L20 Kamiński, T., Schmidt, M., Tylenda, R., et al. 2009, ApJS, 182, 33 Kankare, E., Kotak, R., Pastorello, A., et al. 2015, A\&A, 581, L4 Kasliwal, M. M. 2012, PASA, 29, 482

Kasliwal, M. M., Kulkarni, S. R., Arcavi, I., et al. 2011, ApJ, 730, 134 Kochanek, C. S., Adams, S. M., \& Belczynski, K. 2014, MNRAS, 443, 1319 Kulkarni, S. R., Ofek, E. O., Rau, A., et al. 2007, Nature, 447, 458

Lipunov, V. M., Blinnikov, S., Gorbovskoy, E., et al. 2017, MNRAS, 470, 2339

LSST Science Collaboration 2009, ArXiv e-prints [arXiv: 0912.0201]

MacLeod, M., Macias, P., Ramirez-Ruiz, E., et al. 2017, APJ, 835, 282

Mallik, S. V. 1997, A\&AS, 124, 359

Mason, E., Diaz, M., Williams, R. E., et al. 2010, A\&A, 516, A108

Mauerhan, J. C., Van Dyk, S. D., Johansson, J., et al. 2018, MNRAS, 473, 3765

Mould, J. R., Huchra, J. P., Freedman, W. L., et al. 2000, ApJ, 545, 547

Munari, U., Henden, A., Kiyota, S., et al. 2002, A\&A, 389, L51

Pastorello, A., \& Fraser, M. 2019, Nat. Astron., 3, 676

Pastorello, A., Zampieri, L., Turatto, M., et al. 2004, MNRAS, 347, 74

Pastorello, A., Della Valle, M., Smartt, S. J., et al. 2007, Nature, 449, 1

Pastorello, A., Chen, T. W., Cai, Y. Z., et al. 2019a, A\&A, 625, L8

Pastorello, A., Mason, E., Taubenberger, S., et al. 2019b, A\&A, 630, A75

Pejcha, O., Metzger, B. D., \& Tomida, K. 2016, MNRAS, 461, 2527

Pejcha, O., Metzger, B. D., Tyles, J. G., \& Tomida, K. 2017, ApJ, 850, 59

Pumo, M. L., Turatto, M., Botticella, M. T., et al. 2009, ApJ, 705, L138

Rau, A., Kulkarni, S. R., Ofek, E. O., \& Yan, L. 2007, ApJ, 659, 1536

Schlafly, E. F., \& Finkbeiner, D. P. 2011, ApJ, 737, 103

Smith, N., Andrews, J. E., Van Dyk, S. D., et al. 2016, MNRAS, 458, 950

Smith, K. W., Williams, R. D., Young, D. R., et al. 2019, Res. Notes AAS, 3,26

Spergel, D., Gehrels, N., Baltay, C., et al. 2015, ArXiv e-prints [arXiv: 1503.03757]

Thompson, T. A., Prieto, J. L., Stanek, K. Z., et al. 2009, ApJ, 705, 1364

Tully, R. B., Rizzi, L., Shaya, E. J., et al. 2009, AJ, 138, 323

Turatto, M., Benetti, S., \& Cappellaro, E. 2003, in From Twilight to Highlight -

The Physics of Supernovae, Proc. ESO/MPA/MPE Workshop (Springer), 200

Tylenda, R. 2005, A\&A, 436, 1009

Tylenda, R., Hajduk, M., Kamiński, T., et al. 2011, A\&A, 528, A114

Williams, S. C., Darnley, M. J., Bode, M. F., \& Steele, I. A. 2015, ApJ, 805, L18

Willick, J. A., Courteau, S., Faber, S. M., et al. 1997, ApJS, 109, 333
1 Università degli Studi di Padova, Dipartimento di Fisica e Astronomia, Vicolo dell'Osservatorio 2, 35122 Padova, Italy e-mail: yongzhi.cai@studenti.unipd.it

2 INAF-Osservatorio Astronomico di Padova, Vicolo dell'Osservatorio 5, 35122 Padova, Italy

3 School of Physics, O'Brien Centre for Science North, University College Dublin, Belfield, Dublin 4, Ireland

${ }^{4}$ School of Physics, Trinity College Dublin, The University of Dublin, Dublin 2, Ireland

5 Department of Physics and Astronomy, University of Turku, 20014 Turku, Finland

${ }^{6}$ Department of Applied Physics, University of Cádiz, Campus of Puerto Real, 11510 Cádiz, Spain

7 Millennium Institute of Astrophysics (MAS), Nuncio Monsenor Sòtero Sanz 100, Providencia, Santiago, Chile

8 Departamento de Ciencias Fisicas, Universidad Andres Bello, Fernandez Concha 700, Las Condes, Santiago, Chile

9 SRON, Netherlands Institute for Space Research, Sorbonnelaan, 2, 3584 CA Utrecht, Netherlands

10 Department of Astrophysics/IMAPP, Radboud University, PO Box 9010, 6500 GL Nijmegen, The Netherlands

11 Astronomical Observatory, University of Warsaw, Al. Ujazdowskie 4, 00-478 Warszawa, Poland

12 Nordic Optical Telescope, Apartado 474, 38700 Santa Cruz de La Palma, Spain

13 Depto. de Astrofísica, Centro de Astrobiología (INTA-CSIC), Camino Bajo del Castillo s/n, 28692 Madrid, Spain

14 DARK, Niels Bohr Institute, Lyngbyvej 2, 2100 Copenhagen, Denmark

15 INAF-Osservatorio Astronomico di Trieste, Via Giambattista Tiepolo 11, 34143 Trieste, Italy

16 Istituto di Astrofisica e Planetologia Spaziali (INAF), via del Fosso del Cavaliere 100, Roma 00133, Italy

17 The Oskar Klein Centre, Physics Department, Stockholm University, 10691 Stockholm, Sweden 


\section{Appendix A: Additional information}

\section{HST alignment and measurement details}

NGC 3729 was observed on 2004 November 17 with HST+ACS Unfortunately, these observations were quite shallow, consisting of $2 \times 350 \mathrm{~s}$ in F658N, and $120 \mathrm{~s}$ in F814W. In order to locate the position of the $\mathrm{SN}$ on these images, we aligned the drizzled F814W HST image to the NOT+ALFOSC $i$-band image taken on 2019 January 14. Only five sources were used for the alignment, which has a root mean square (RMS) scatter of $0.12^{\prime \prime}$. In light of the small number of fiducial sources for the alignment, we caution that there could be a larger systematic uncertainty in position. A bright source is detected with an $S / N \approx 17$, within $2 \sigma$ of the transformed SN position (see Fig. A.1). From the density of sources close to the location of AT 2018hso in the HST images, we calculate that the probability of a chance alignment with a source as bright as the progenitor candidate is $6 \%$. Using DOLPHOT, we measure F658N $=23.29 \pm 0.16 \mathrm{mag}$ and $\mathrm{F} 814 \mathrm{~W}=23.56 \pm 0.07 \mathrm{mag}$ (Vegamag, in the HST system) for the object. At the adopted distance, we obtain an absolute magnitude of $M_{\mathrm{I}} \sim-8 \mathrm{mag}$.

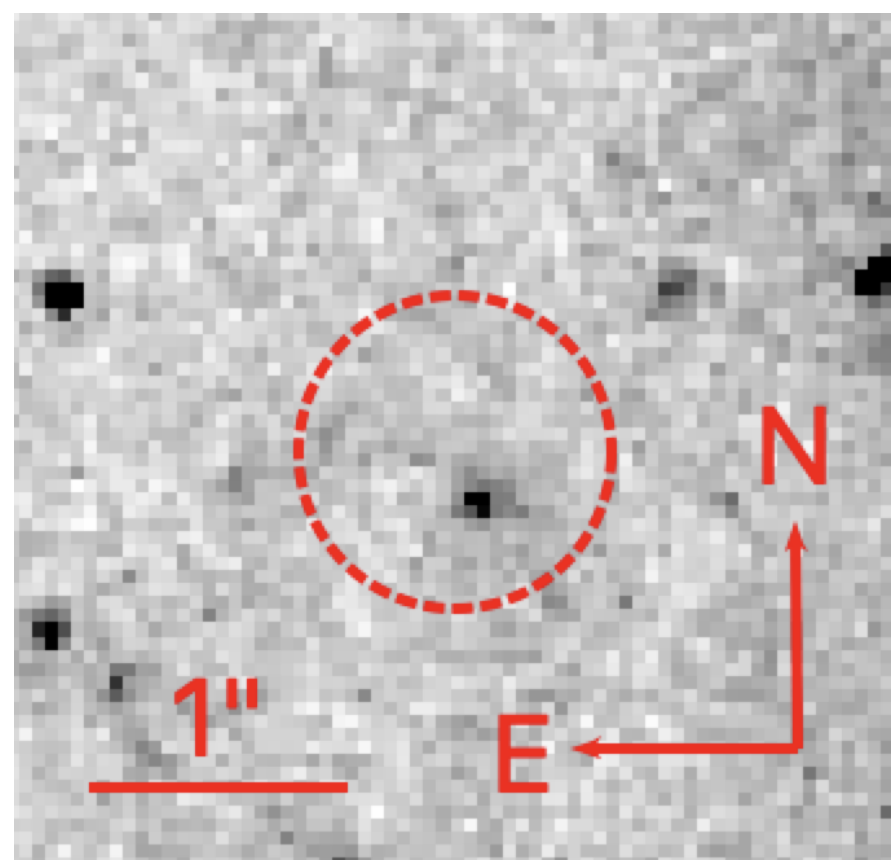

Fig. A.1. Pre-outburst archive image of AT 2018hso obtained with HST+F814W. The red dashed circle shows the 5x RMS (0.6") uncertainty in the position. 
Table A.1. Optical (BVugriz) light curves of AT 2018hso.

\begin{tabular}{|c|c|c|c|c|c|c|c|c|c|}
\hline Date & MJD & $B(\mathrm{err})$ & $V(\mathrm{err})$ & $u(\mathrm{err})$ & $g(\mathrm{err})$ & $r(\mathrm{err})$ & $i(\mathrm{err})$ & $z(\mathrm{err})$ & Instrument \\
\hline 20181031 & 58422.487 & - & - & - & $>19.5$ & - & - & - & (1) \\
\hline 20181031 & 58423.036 & - & - & - & - & $19.410(0.3)$ & - & - & (1) \\
\hline 20181101 & 58423.466 & - & - & - & $>19.7$ & - & - & - & (1) \\
\hline 20181101 & 58423.534 & - & - & - & - & $>19.9$ & - & - & (1) \\
\hline 20181102 & 58424.529 & - & - & - & - & $18.920(0.3)$ & - & - & (1) \\
\hline 20181104 & 58426.487 & - & - & - & $19.103(0.096)$ & - & - & - & (1) \\
\hline 20181104 & 58426.530 & - & - & - & - & $18.656(0.081)$ & - & - & (1) \\
\hline 20181106 & 58428.270 & $19.310(0.047)$ & $18.926(0.040)$ & $19.876(0.119)$ & $19.088(0.042)$ & $18.627(0.035)$ & $18.638(0.044)$ & $18.508(0.053)$ & (2) \\
\hline 20181107 & 58429.469 & - & - & - & $19.259(0.115)$ & - & - & - & (1) \\
\hline 20181110 & 58432.456 & - & - & - & $18.960(0.088)$ & - & - & - & (1) \\
\hline 20181110 & 58432.547 & - & - & - & - & $18.483(0.066)$ & - & - & (1) \\
\hline 20181115 & 58437.210 & $19.864(0.030)$ & $19.206(0.039)$ & - & - & $18.612(0.114)$ & $18.666(0.054)$ & - & (3) \\
\hline 20181116 & 58438.210 & $19.838(0.154)$ & $19.321(0.070)$ & $20.147(0.157)$ & $19.236(0.050)$ & $18.895(0.037)$ & $18.665(0.039)$ & $18.671(0.056)$ & (2) \\
\hline 20181118 & 58440.530 & - & - & - & - & $19.025(0.078)$ & - & - & (1) \\
\hline 20181120 & 58442.215 & $20.212(0.088)$ & $19.378(0.081)$ & $20.709(0.089)$ & $19.777(0.031)$ & $19.285(0.040)$ & $18.870(0.059)$ & $18.971(0.171)$ & (3) \\
\hline 20181121 & 58443.431 & - & - & - & - & $19.291(0.168)$ & - & - & (1) \\
\hline 20181125 & 58447.270 & - & - & $>21.0$ & $20.352(0.120)$ & $19.378(0.058)$ & $19.262(0.091)$ & $19.130(0.116)$ & (2) \\
\hline 20181125 & 58447.470 & - & - & - & - & $19.620(0.186)$ & - & - & (1) \\
\hline 20181201 & 58453.240 & $20.947(0.105)$ & $19.955(0.109)$ & $22.482(0.148)$ & $20.509(0.069)$ & $19.659(0.077)$ & $19.529(0.057)$ & $19.254(0.042)$ & (3) \\
\hline 20181204 & 58456.175 & - & - & - & $20.638(0.101)$ & $19.735(0.059)$ & $19.582(0.113)$ & $19.276(0.058)$ & (2) \\
\hline 20181210 & 58462.190 & - & - & - & $21.082(0.089)$ & $19.844(0.043)$ & $19.627(0.026)$ & $19.248(0.032)$ & (3) \\
\hline 20181212 & 58464.180 & - & - & - & $21.126(0.132)$ & $19.807(0.062)$ & $19.546(0.071)$ & $19.164(0.093)$ & (2) \\
\hline 20181216 & 58468.175 & - & - & - & $21.233(0.111)$ & $19.783(0.057)$ & $19.423(0.054)$ & $19.068(0.079)$ & (2) \\
\hline 20181220 & 58472.110 & - & - & - & $>21.3$ & $19.746(0.062)$ & $19.407(0.044)$ & - & (2) \\
\hline 20181220 & 58472.550 & - & - & - & - & $19.811(0.194)$ & - & - & (1) \\
\hline 20181226 & 58478.135 & - & - & - & $21.637(0.267)$ & $19.702(0.073)$ & $19.337(0.053)$ & $18.883(0.131)$ & (2) \\
\hline 20181229 & 58481.135 & $22.168(0.229)$ & $20.475(0.099)$ & - & - & - & - & - & (3) \\
\hline 20181230 & 58482.115 & - & - & - & $21.690(0.120)$ & $19.649(0.075)$ & $19.307(0.032)$ & $18.861(0.086)$ & (2) \\
\hline 20190103 & 58486.225 & $22.381(0.171)$ & $20.528(0.048)$ & - & - & - & - & - & (3) \\
\hline 20190106 & 58489.085 & - & - & - & $21.908(0.180)$ & $19.644(0.107)$ & $19.308(0.042)$ & $18.878(0.070)$ & (2) \\
\hline 20190108 & 58491.557 & - & - & - & - & $19.698(0.130)$ & - & - & (1) \\
\hline 20190110 & 58493.235 & $22.544(0.142)$ & $20.608(0.045)$ & - & $21.861(0.100)$ & $19.607(0.079)$ & $19.284(0.063)$ & $18.772(0.048)$ & (3) \\
\hline 20190114 & 58497.175 & $22.559(0.134)$ & $20.644(0.085)$ & - & $21.843(0.084)$ & $19.567(0.113)$ & $19.268(0.113)$ & $18.926(0.067)$ & (3) \\
\hline 20190120 & 58503.175 & $22.594(0.195)$ & $20.543(0.040)$ & - & $21.788(0.154)$ & $19.510(0.110)$ & $19.156(0.054)$ & $18.875(0.034)$ & (3) \\
\hline 20190125 & 58508.484 & - & - & - & - & $19.543(0.178)$ & - & - & (1) \\
\hline 20190128 & 58511.386 & - & - & - & - & $19.516(0.122)$ & - & - & (1) \\
\hline 20190206 & 58520.145 & - & $20.494(0.043)$ & - & $21.657(0.074)$ & $19.672(0.026)$ & $19.091(0.020)$ & $18.737(0.040)$ & (3) \\
\hline 20190212 & 58526.175 & $22.479(0.197)$ & $20.392(0.059)$ & - & $21.637(0.068)$ & $19.632(0.041)$ & $19.093(0.013)$ & $18.773(0.014)$ & (3) \\
\hline 20190221 & 58535.095 & $22.529(0.223)$ & $20.452(0.051)$ & - & $21.767(0.366)$ & $19.869(0.051)$ & $19.146(0.040)$ & $18.715(0.040)$ & (3) \\
\hline 20190227 & 58541.995 & $22.824(0.249)$ & $20.580(0.049)$ & - & $21.968(0.115)$ & $19.900(0.032)$ & $19.258(0.020)$ & $18.832(0.038)$ & (3) \\
\hline 20190315 & 58557.085 & $>22.9$ & $21.064(0.124)$ & - & $>22.2$ & $20.288(0.051)$ & $19.591(0.037)$ & $18.887(0.048)$ & (3) \\
\hline 20190318 & 58560.875 & - & - & - & $>21.9$ & $20.358(0.139)$ & $19.675(0.079)$ & $18.983(0.073)$ & (2) \\
\hline 20190323 & 58565.950 & - & - & - & - & $20.512(0.095)$ & $19.659(0.051)$ & - & (2) \\
\hline 20190407 & 58580.995 & - & - & - & $22.790(0.200)$ & $20.631(0.138)$ & $19.712(0.045)$ & $19.120(0.073)$ & (3) \\
\hline 20190408 & 58581.005 & $>23.5$ & $21.469(0.075)$ & - & - & - & - & - & (3) \\
\hline 20190416 & 58589.080 & - & $>21.6$ & - & $>22.5$ & $20.835(0.131)$ & $19.835(0.043)$ & $19.133(0.057)$ & (3) \\
\hline 20190420 & 58593.930 & - & $21.755(0.204)$ & - & $22.936(0.221)$ & $20.918(0.284)$ & $19.863(0.290)$ & $19.267(0.059)$ & (3) \\
\hline 20190428 & 58601.950 & - & $22.061(0.211)$ & - & - & $21.060(0.086)$ & $19.932(0.033)$ & $19.202(0.036)$ & (3) \\
\hline 20190512 & 58615.890 & - & $22.249(0.176)$ & - & $>23.0$ & $21.126(0.090)$ & $19.982(0.036)$ & $19.279(0.032)$ & (3) \\
\hline 20190530 & 58633.885 & - & $22.819(0.319)$ & - & $>23.1$ & $21.577(0.119)$ & $20.326(0.044)$ & $19.528(0.043)$ & (3) \\
\hline 20190611 & 58645.925 & - & $>23.0$ & - & - & $22.007(0.073)$ & $21.001(0.054)$ & $19.750(0.054)$ & (3) \\
\hline 20190617 & 58651.945 & - & - & - & - & $22.497(0.183)$ & $21.269(0.051)$ & $20.203(0.052)$ & (3) \\
\hline 20190629 & 58663.880 & - & - & - & - & - & $21.544(0.118)$ & - & (4) \\
\hline 20190702 & 58666.895 & - & - & - & - & $23.172(0.521)$ & - & $20.433(0.058)$ & (3) \\
\hline 20190716 & 58680.915 & - & - & - & - & - & $21.952(0.243)$ & $20.705(0.091)$ & (3) \\
\hline 20190803 & 58698.875 & - & - & - & - & - & $>22.4$ & $21.216(0.127)$ & (3) \\
\hline
\end{tabular}

Notes. ${ }^{(1)}$ ZTF (https://www.ztf.caltech.edu/) data from the Palomar $1.2 \mathrm{~m}$ Oschin Telescope equipped with ZTFCam, taken from LASAIR (https://lasair.roe.ac.uk/object/ZTF18acbwfza/) (Smith et al. 2019) and Transient Name Server (TNS, https://wis-tns.weizmann.ac.il/object/2018hso). ${ }^{(2)}$ The $2 \mathrm{~m}$ fully automatic Liverpool Telescope (LT, http://telescope.livjm.ac.uk/) equipped with IO:O, located at Roque de los Muchachos Observatory (La Palma, Canary Islands, Spain). ${ }^{(3)}$ The $2.56 \mathrm{~m}$ Nordic Optical Telescope (NOT, http: //www . not.iac. es/) equipped with ALFOSC, located at Roque de los Muchachos Observatory (La Palma, Canary Islands, Spain). ${ }^{(4)}$ The $10.4 \mathrm{~m}$ Gran Telescopio Canarias (GTC, http://www.gtc.iac.es/) with OSIRIS, located at Roque de los Muchachos Observatory (La Palma, Canary Islands, Spain). 
Table A.2. NIR $(J H K)$ light curves of AT 2018hso.

\begin{tabular}{lccccc}
\hline \hline Date & MJD & $J($ err $)$ & $H($ err $)$ & $K($ err $)$ & Instrument \\
\hline 20181104 & 58426.217 & $17.462(0.034)$ & $17.074(0.024)$ & $16.332(0.026)$ & NOTCAM \\
20181124 & 58446.150 & $18.143(0.091)$ & - & - & NOTCAM \\
20181211 & 58463.153 & $17.890(0.104)$ & $17.641(0.119)$ & $16.877(0.126)$ & NOTCAM \\
20181231 & 58483.070 & $17.585(0.117)$ & $17.329(0.115)$ & $16.725(0.111)$ & NOTCAM \\
20190111 & 58494.160 & $17.673(0.098)$ & $17.131(0.098)$ & $16.560(0.163)$ & NOTCAM \\
20190130 & 58513.213 & $17.598(0.097)$ & $17.014(0.102)$ & $16.506(0.125)$ & NOTCAM \\
20190305 & 58547.080 & $17.424(0.092)$ & $16.782(0.093)$ & $16.060(0.119)$ & NOTCAM \\
20190322 & 58564.045 & $17.518(0.122)$ & - & $16.301(0.106)$ & NOTCAM \\
20190410 & 58583.053 & $17.693(0.035)$ & $17.053(0.124)$ & $16.106(0.114)$ & NOTCAM \\
20190430 & 58603.950 & $17.599(0.074)$ & $16.933(0.096)$ & $15.991(0.547)$ & NOTCAM \\
20190613 & 58647.970 & $18.091(0.122)$ & $17.666(0.119)$ & $16.437(0.121)$ & NOTCAM \\
20190703 & 58667.933 & $18.430(0.098)$ & $18.030(0.064)$ & $16.833(0.155)$ & NOTCAM \\
\hline
\end{tabular}

Notes. NOTCAM: The $2.56 \mathrm{~m}$ Nordic Optical Telescope (NOT) equipped with NOTCAM, located at Roque de los Muchachos Observatory (La Palma, Canary Islands, Spain).

Table A.3. General information about the spectroscopic observations of AT 2018hso.

\begin{tabular}{|c|c|c|c|c|c|c|c|}
\hline Date & MJD & $\begin{array}{c}\text { Phase }^{(a)} \\
\text { (days) }^{\text {days }}\end{array}$ & Telescope+Instrument & Grism+Slit & $\begin{array}{c}\text { Spectral range } \\
(\AA)\end{array}$ & $\begin{array}{c}\text { Resolution } \\
(\AA)\end{array}$ & $\begin{array}{c}\text { Exp. time } \\
\text { (s) }\end{array}$ \\
\hline 20181105 & 58427.2 & -3.8 & $\mathrm{NOT}+\mathrm{ALFOSC}$ & $\mathrm{gm} 4+1.0^{\prime \prime}$ & $3400-9650$ & 15 & 1670 \\
\hline 20181120 & 58442.3 & +11.3 & $\mathrm{NOT}+\mathrm{ALFOSC}$ & $\mathrm{gm} 4+1.0^{\prime \prime}$ & $3500-9630$ & 15 & 3600 \\
\hline 20181210 & 58462.2 & +31.2 & $\mathrm{NOT}+\mathrm{ALFOSC}$ & $\mathrm{gm} 4+1.0^{\prime \prime}$ & 3400-9600 & 15 & 3600 \\
\hline 20181212 & 58464.3 & +33.3 & $\mathrm{NOT}+\mathrm{ALFOSC}$ & gm4+1.3" & $3400-9600$ & 18 & 3600 \\
\hline 20181229 & 58481.2 & +50.2 & $\mathrm{NOT}+\mathrm{ALFOSC}$ & $\mathrm{gm} 4+1.0^{\prime \prime}$ & $3400-9600$ & 15 & 3600 \\
\hline 20190123 & 58506.2 & +75.2 & $\mathrm{NOT}+\mathrm{ALFOSC}$ & $\mathrm{gm} 4+1.0^{\prime \prime}$ & $3400-9650$ & 15 & 3600 \\
\hline 20190228 & 58542.0 & +111.0 & $\mathrm{NOT}+\mathrm{ALFOSC}$ & $\mathrm{gm} 4+1.0^{\prime \prime}$ & $3400-9650$ & 15 & 3600 \\
\hline 20190315 & 58557.1 & +126.1 & $\mathrm{NOT}+\mathrm{ALFOSC}$ & $\mathrm{gm} 4+1.0^{\prime \prime}$ & $3400-9650$ & 15 & 3600 \\
\hline 20190409 & 58582.0 & +151.0 & $\mathrm{NOT}+\mathrm{ALFOSC}$ & $\mathrm{gm} 4+1.0^{\prime \prime}$ & $4000-9600$ & 15 & 3600 \\
\hline 20190428 & 58602.0 & +171.0 & $\mathrm{NOT}+\mathrm{ALFOSC}$ & $\mathrm{gm} 4+1.0^{\prime \prime}$ & $3600-9600$ & 15 & 3600 \\
\hline 20190629 & 58663.9 & +232.9 & GTC+OSIRIS & $\mathrm{R} 1000 \mathrm{R}+1.0^{\prime \prime}$ & $5100-10350$ & 8 & $3 \times 1800$ \\
\hline
\end{tabular}

Notes. ${ }^{(a)}$ Phases are relative to the $r$-band maximum $\left(\mathrm{MJD}_{\mathrm{AT} 2018 \mathrm{hso}}=58431.0 \pm 1.0\right)$. 